\title{
Atypical and Conventional Antipsychotic Drugs in Treatment- Naive First-Episode Schizophrenia: A 52-Week Randomized Trial of Clozapine Vs Chlorpromazine
}

\author{
Jeffrey A Lieberman*,', Michael Phillips ${ }^{3}$, Hongbin Gu', Scott Stroup', Peiyan Zhang ${ }^{3}$, Lan Kong², \\ Zhongfu $\mathrm{Ji}^{3}$, Gary Koch ${ }^{2}$ and Robert M Hamer ${ }^{1,2}$ \\ 'Department of Psychiatry, University of North Carolina School of Medicine Chapel Hill, North Carolina, USA; ${ }^{2}$ Department of Biostatistics, \\ University of North Carolina School of Medicine Chapel Hill, North Carolina, USA; ${ }^{3}$ Beijing Huilonguan Hospital, Beijing, Peoples Republic of China
}

\begin{abstract}
The purported advantages of second-generation or 'atypical' antipsychotics relative to first-generation antipsychotics have not been examined in patients with a first episode of schizophrenia. This flexible-dose study examined efficacy and safety in a randomized, doubleblind, 52-week trial, comparing chlorpromazine (CPZ) and clozapine (CLZ) in treatment naive patients experiencing their first episode of schizophrenia. In all, 160 inpatients with first-episode schizophrenia or schizophreniform disorder were randomized to CPZ or CLZ and followed them for 52 weeks or until dropout. The primary efficacy measure was time to first remission and proportion of time remaining in remission. The analysis was supplemented by comparisons on a profile of clinical symptoms and side effects. Of these first-episode patients, $80 \%$ achieved remission within I year (79\% CPZ, 81\% CLZ). The Kaplan-Meier estimated median time to first remission was 8 weeks for $C L Z$ vs 12 weeks for CPZ $\left(\chi^{2}(1)=5.56, p=0.02\right)$. Both the rate of first achieving remission and the odds for being in remission during the trial were almost doubled for the CLZ group in comparison with the CPZ group. At 12 weeks, CLZ was superior on many rating scale measures of symptom severity while CPZ was not superior on any. These symptom differences remained significant when controlling for EPS differences. By 52 weeks many of the symptom differences between groups were no longer significantly different. Generally, CLZ produced fewer side effects than CPZ, particularly extrapyramidal side effects. There was no significant difference between treatments in weight change or glucose metabolism. For each prior year of untreated psychosis, there was a $15 \%$ decrease in the odds of achieving remission ( $O R=0.85 ; \mathrm{Cl} 0.75-0.95)$. A high proportion of first-episode patients remitted within I year. We detected no difference in the proportion of first-episode patients receiving CLZ or CPZ that achieved remission. However, firstepisode patients receiving CLZ remitted significantly faster and remained in remission longer than subjects receiving CPZ. While the CLZ group showed significantly less symptomatology on some measures and fewer side effects at 12 weeks, the two treatment groups seemed to converge by I year. Longer duration of untreated psychosis was associated with lower odds of achieving remission. Neuropsychopharmacology (2003) 28, 995-1003, advance online publication, 26 March 2003; doi: I0.1038/sj.npp. I 300 I57
\end{abstract}

Keywords: first-episode schizophrenia; clozapine; chlorpromazine; China; randomized controlled trial; longitudinal

\section{INTRODUCTION}

Historically, the prognoses of patients diagnosed with schizophrenia were considered variable and pessimistic (Kraeplin, 1919). This is because schizophrenia was believed to be a brain disorder that was expressed in abnormal mental functions, which characteristically appeared in the late second and third decades of life, and usually led to a

*Correspondence: Dr JA Lieberman, Department of Psychiatry, C.B. \#7160, University of North Carolina, Chapel Hill, NC 27599-7160, USA, Tel: + I 9199668990 , Fax: + I 9199668994 ,

E-mail: jlieberman@med.unc.edu

Received 29 August 2002; revised 20 December 2002; accepted 02 January 2003

Online publication: 08 January 2002 at http://www.acnp.org/citations/ Npp0 0803452 chronic or recurrent course that produced clinical deterioration in the form of persistent symptoms, impairments in social and work functions, and erosion of the personality (McGlashan, 1988).

Following their introduction in the 1950s, conventional antipsychotic medications, or neuroleptics, were shown to suppress the acute psychotic symptoms of the disorder and prevent their recurrence. However, many patients were found to be unresponsive to neuroleptics and it was generally believed that, despite their ability to suppress symptoms, they did not change the long-term course of the disorder or improve the outcome (Hegarty et al, 1994). In addition, neuroleptics were associated with high rates of neurological side effects (ie acute extrapyramidal signs (EPS) and tardive dyskinesia (TD)), which compromised the therapeutic effects of treatment and impelled many 
patients to discontinue treatment, which led to higher relapse rates.

In contrast, studies of patients in the first episode of their illness demonstrated high rates of therapeutic response and symptomatic remission in comparison with chronic patients with multiple prior episodes (Lieberman et al, 1993, 1998; Sheitman et al, 1997; Lieberman et al, 1998). At the same time, they also experienced high rates of neurological side effects to neuroleptic drugs (Chatterjee et al, 1995; Chakos et al, 1996). In general, first-episode patients appeared to be more sensitive to the pharmacologic effects of antipsychotic drugs than older chronic patients in that they exhibited higher rates of recovery, more frequent extrapyramidal side effects, and required lower drug doses (Lieberman et al, 1998; McEvoy et al, 1991). However, following their recovery first-episode patients experienced a high rate of psychotic relapse particularly if they discontinued antipsychotic medication (Crow et al, 1986; Robinson et al, 1999).

The advent of the second-generation or so-called 'atypical' antipsychotic drugs was heralded as a therapeutic advance in the treatment of schizophrenia and related psychotic disorders. It was claimed that atypical antipsychotics had a different mechanism of action and were more effective and safer than conventional antipsychotics (Kinon and Lieberman, 1996). However, these claims are only partially supported by empirical data, and there is a substantial variation in opinion among researchers and clinicians about their comparative effectiveness (Leucht et al, 1999; Geddes et al, 2000; Chakos et al, 2001). Since their introduction into clinical practice, atypical antipsychotic drugs have been examined in comparison with the conventional antipsychotics in heterogeneous patient samples predominantly comprising chronically ill patients whose symptoms may have been (partially) resistant to treatment. The question of the comparative efficacy and safety of these two classes of antipsychotic drugs has not been examined in first-episode patients beyond short-term trials (Emsley, 1999; Sanger et al, 1999). Indeed this question has considerable importance, since first-episode patients are highly responsive to pharmacologic treatment, and the first treatment intervention in drug naive patients is a critical therapeutic opportunity that has the potential to influence the course and outcome of what could be a lifelong illness (Lieberman, 1996). This is particularly the case because longer duration in the first episode of psychosis has been associated with poorer treatment response and outcome (Norman and Malla, 2001). Thus, the rapidity and maintenance of treatment response, as well as tolerability, which is associated with treatment adherence, may be important factors in determining the outcome.

Our hypothesis was that clozapine (CLZ), the prototypical second-generation antipsychotic, had superior efficacy in first-episode patients when compared to a representative conventional antipsychotic. When this study was conceived, CLZ was the only second-generation antipsychotic marketed in any part of the world. By the mid-1990s, CLZ was, depending on the location, the first or second most commonly used antipsychotic in China ( $\mathrm{Wu}$ and $\mathrm{Wu}$, 1995; Phillips, 2001). Although in most Western countries CLZ is used as a second-line treatment because of its hematological toxicity (Herz et al, 1997), in China it is often used in the first episode of illness. Given this set of circumstances, we felt it was experimentally feasible and clinically justifiable to examine the efficacy of CLZ in firstepisode patients in China.

\section{METHODS}

\section{Setting}

The study was conducted at the Beijing Hui Long Guan Hospital, one of the largest psychiatric hospitals in China, which is located $30 \mathrm{~km}$ from central Beijing and serves a catchment area population of 13 million people. Dr Phillips has worked in the PRC since 1985 and is the Director of the Research Center of Clinical Epidemiology at the Hui Long Guan Hospital. The study protocol was designed by the first and second authors (JL and MP). It conformed to international standards for research ethics and was approved by the Research Review Board of the Beijing Bureau of Health prior to its initiation and monitored annually while in progress. Funding for the study and medication supplies were provided by the Novartis Pharmaceutical Company. The authors had unrestricted access to the data; company personnel were not involved in the analysis of the data nor did they have any authority over publication.

\section{Subjects}

All hospital admissions were screened from October 1995 to December 1998 for patients who met the following criteria: (1) diagnosis of schizophrenia or schizophreniform disorder; (2) duration of symptoms not longer than 60 months; (3) no prior treatment with antipsychotic medication or, if previously treated, a total lifetime usage of less than 14 days; (4) between 16 and 40 years of age; and (5) current psychotic symptoms of moderate severity or greater measured by one of the five psychotic items in the Brief Psychiatric Rating Scale (BPRS). Patients meeting these criteria, and their families, provided written informed consent to participate.

\section{Procedures}

Patients' diagnoses were based on the results of the Structured Clinical Interview for DSM (SCID) using the criteria of the fourth edition of the Diagnostic and Statistical Manual (DSM-IV), physical exams and routine chemistry, and hematology laboratory tests. Attending-level psychiatrists used a Chinese translation of the SCID to interview patients and their family members, and were given freedom to ask additional questions if respondents did not understand the standard probes. There are no major difficulties in employing DSM IV criteria to diagnose schizophrenia in China; current Chinese diagnostic criteria for schizophrenia are similar to ICD-10 criteria, but many locations also use DSM IV criteria. Patients were randomized to treatment with CLZ plus benztropine (BZ) placebo or chlorpromazine $(\mathrm{CPZ})$ and $\mathrm{BZ}$ under double-blind conditions. Medication dosages were increased using a standardized regimen during the first 28 days of admission (to a maximum of $400 \mathrm{mg} /$ day of CLZ or $600 \mathrm{mg} /$ day of CPZ) and then adjusted 
based on the clinical response and side effects. Patients taking $\mathrm{CPZ}$ were also given $2 \mathrm{mg} \mathrm{BZ}$ twice a day and those on CLZ were given the corresponding placebos. Patients received inpatient care for 12 weeks (a typical length of stay in Chinese psychiatric hospitals), and were then followed as outpatients on a monthly basis for the subsequent 9 months. After the initial 12-month study period, patients were eligible to enter a double-blind extension phase of the study that is still under way.

Patients dropped out of the study because of withdrawal of consent by patients or family members, failure of treatment to relieve symptoms, or severe side effects. Detailed treatment adherence information was recorded; all patients in the study continued the clinical and side effect evaluations as per schedule even if noncompliance occurred, in order to ensure the completeness of data on the total sample.

\section{Assessments}

Patients were evaluated using the Chinese version of the BPRS (Phillips et al, 1990), the Chinese version of the Scale for Assessment of Negative Symptoms (SANS), (Phillips et al, 1991), Clinical Global Impression Scale (CGI), Global Assessment of Function Scale (GAF), the Simpson Angus Extrapyramidal Symptoms Scale (SAESS) (with sialorrhea omitted because of its well-known connection with CLZ) and for side effects at study entry, weekly for weeks 1-6, biweekly for weeks 6-12, at hospital discharge, and at 3monthly intervals following discharge. Side effects were recorded using the Coding Symbol and Thesaurus for Adverse Event Terminology (COSTART) classification terms at each assessment visit. EKGs were performed at study entry, weeks 12 and 52. All patients underwent venipunctures to monitor white blood counts (WBC) weekly while inpatients and monthly after discharge. Serum glucose was assessed under fasting conditions at study entry, week 12 , and every 3 months after discharge. Weight was not measured regularly by the protocol or hospital procedure. Consequently, only subgroups of patients in this trial had baseline and follow-up weights. Moreover, most of the follow-up weights were taken after the 52-week period of the formal trial and were not standardized in terms of frequency and time intervals. Since patients who successfully completed the trial were continued on their study medication in a blinded follow-up phase of the study thereafter, we included the first available follow-up weight of each patient in the analysis $(n=57$ for CPZ and $n=58$ for CLZ).

\section{Statistical Methods}

The primary measure for treatment efficacy is remission, which was defined a priori as a $50 \%$ reduction in total BPRS score from baseline with no score greater than mild (3) on the 5 BPRS psychosis items (unusual thought content, suspiciousness, hallucinations, conceptual disorganization, mannerisms, and posturing) and a CGI-severity item of mild (3) or less. Using an intent-to-treatment (ITT) population, we compared the time to first remission between the two treatments using Kaplan-Meier estimates for their survival curves, and refined the model by controlling for baseline BPRS, gender, duration of untreated psychosis (DUP), and age of onset in Cox proportional hazard regression. Owing to the recurrence of remissions and relapses, we identified the week-by-week remission status for each patient during the first 12 weeks of treatment and then monthly thereafter evaluated the overall remission status by taking the proportion of weeks on remission over the total number of weeks followed for each individual, and modeled it using generalized estimating equation (GEE) logistic regression with robust standard error estimates for the possible overdispersion caused by correlated responses within patients.

For secondary efficacy measures, we looked at the BPRS, SANS, CGI, and GAF scores at the end of inpatient treatment (12 weeks) and at the end of the study (52 weeks). We compared the CPZ and CLZ groups using ANCOVA models controlling for baseline, gender, duration, and age of onset of psychotic symptoms. The possible interactions between treatment and covariates were tested and explored in further detail if significant. The comparison was conducted using both the completers at the time point (observed cases (OC)) and the ITT patient population with missing values filled in from the last available observation (last observation carried forward (LOCF)).

The clinical and laboratory parameters (percentage of polymorphonuclear neutrophils and lymphocytes, WBC, glucose, heart rate, and EKG QT interval) and severity of side effects (total SAESS score and parkinsonism subscale) were compared between the two groups using ANCOVA models similar to those for the continuous efficacy measures. For recurrent adverse events (COSTART terms), we recoded all the ranking scales (0-3) higher than 0 for the presence of the side effects. The transformation from ranking to binary scale was conservative; however, by pooling sparse observations of higher rank responses, we were able to get more reliable estimates of odds ratios. The proportion of visits recorded with occurrence was then modeled for each adverse event using the GEE logistic regression similar to that for remission.

The test on primary outcome used a two-sided alpha-level of 0.05 . Other tests on numerous clinical measures and side effects were conducted as secondary analysis to supplement our major finding. Our focus was on the efficacy and safety profiles of CLZ and CPZ, instead of on any specific score. $p$ Values from the tests for the 14 subscale scores considered were reported and no multiple comparison adjustment was applied.

\section{RESULTS}

\section{Description of Study Cohort}

Of 2708 patients screened for the study, 171 met the criteria for first-episode patients. Of these, 164 patients consented to study participation, 81 were randomized to CLZ, and 83 to CPZ. However, one patient in the CLZ and three in the $\mathrm{CPZ}$ group withdrew before the first administration of medication. Thus, the valid number of patients in each treatment group was 80 (Figure 1).

The two treatment groups were comparable in age, gender, diagnosis, baseline symptom severity, and duration and age of onset of psychotic symptoms. Patients ranged in 


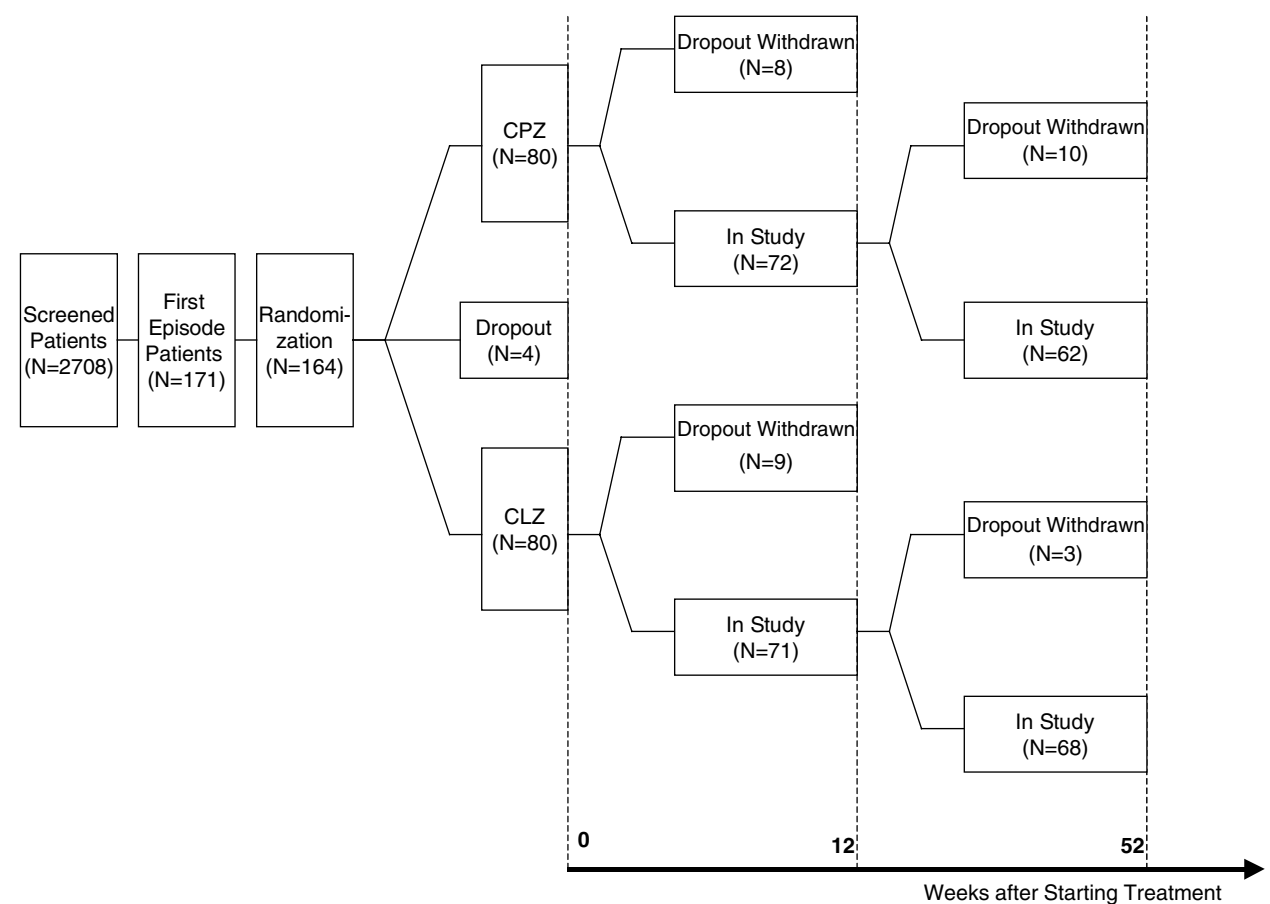

Figure I The design and patient flow of the study. Of the 2708 patients screened for the study, I I I met the inclusion criteria, 164 patients consented to study participation, 81 were randomized to $C L Z$, and 83 to $C P Z$; three patients in the $C L Z$ and one in the CPZ group withdrew before the first administration of medication; so the valid number of patients in each treatment group was 80 . Nine CPZ- and 10 CLZ-treated patients withdrew their consent and dropped out of the study. Three patients were withdrawn for lack of efficacy in the CPZ group. Six CPZ-treated and two CLZ-treated patients were withdrawn for adverse effects. The overall attrition rates at week 12 were $10 \%$ and I I\% for CLZ and CPZ groups, respectively. By 52 weeks, $22.5 \%$ for CPZ and I5\% for CLZ.

age from 15 to 42 (mean[SD], 28.7[6.9]) years; $48 \%$ were female, all were Chinese. (Some patients older than the enrollment criteria were included because their actual age (by their birth certificate) was greater than the age they reported at the time of admission.) Among them $52.5 \%$ were diagnosed with paranoid schizophrenia; $23.1 \%$ with undifferentiated schizophrenia; and $24.4 \%$ with schizophreniform disorder. The first psychotic symptom occurred at the mean age of 27.2[6.5] (median=26.9) in this group of patients. At the time of study entry, patients had had psychotic symptoms for an average of 18.4[17.8] months (median $=10.7)$. At baseline, the average symptom severity score was 43.8[5.1] for total BPRS, 5.6[0.6] for CGI, and 35.8[7.8] for GAF.

The median doses (mg/day) at 12 weeks and at the end of 1-year follow-up were 400 and 300, respectively, for CLZ, and 600 and 400 for CPZ. Nine CPZ- and 10 CLZ-treated patients withdrew their consent and dropped out of the study. Three patients were withdrawn for lack of efficacy in the CPZ group. Six CPZ-treated and two CLZ-treated patients were withdrawn for adverse effects. No severe noncompliance occurred during the acute inpatient phase, but 26 of $72(36 \%) \mathrm{CPZ}$ and 23 of 71 (32\%) CLZ patients took less than $50 \%$ of their prescribed medications, as determined by pill counts, for more than 1 month after discharge. The overall attrition rates were similar at week 12 for $\mathrm{CPZ}$ and CLZ groups at $10 \%$ and $11 \%$, respectively. By the end of study, more patients had dropped out or been withdrawn in CPZ $(22.5 \%)$ than in CLZ (15\%) group, but this difference was not statistically significant (Figure 1).

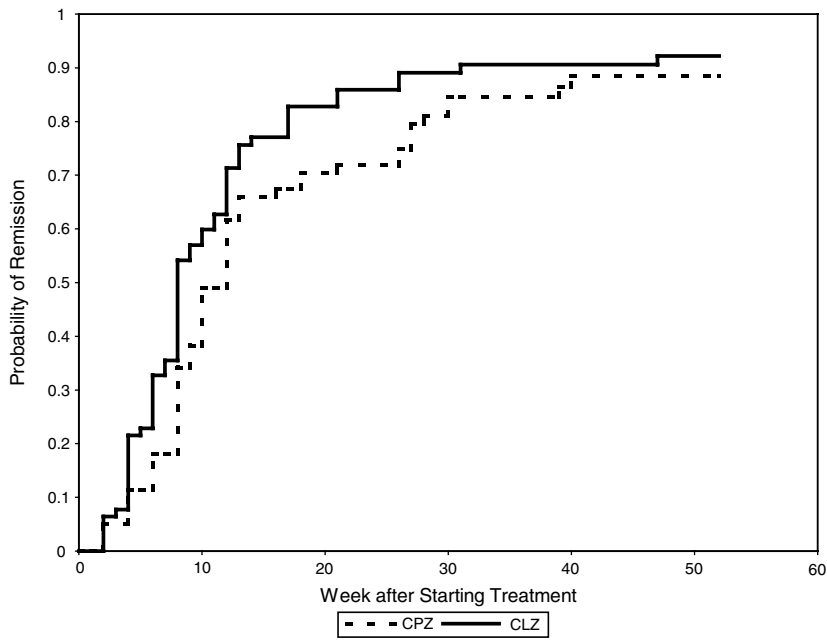

Figure 2 Kaplan-Meier remission survival plots for time to first remission for CPZ (broken line) and CLZ (solid line) groups. The median time to remission in the CLZ group was 8 and 12 weeks in the CPZ group.

\section{Efficacy}

Remission. In all, $79 \%$ of patients $(63 / 80)$ on $\mathrm{CPZ}$ and $81 \%$ $(65 / 80)$ on CLZ had remitted before leaving or completing 52 weeks in the study. Figure 2 shows the Kaplan-Meier remission survival plots for time to remission for $\mathrm{CPZ}$ and CLZ groups. Patients in the CLZ group achieved their remission faster than patients in the $\mathrm{CPZ}$ group 
$\left(\chi^{2}(1)=4.10, p=0.04\right.$ by Log-Rank test). It took 8 weeks of treatment for half the patients in the CLZ group to achieve their first remission compared to 12 weeks in the CPZ group $\left(\chi^{2}(1)=5.56, p=0.02\right.$ by Wilcoxon test). The Cox-regression model adjusting for baseline total BPRS, patient sex, DUP, and age of first psychotic symptoms found that the remission rate was almost twice as fast in the CLZ group as in the $\mathrm{CPZ}$ group (hazard ratio $=1.69,95 \% \mathrm{CI}(1.18,2.44)$, $p=0.005$ ).

The GEE logistic regression model found that patients in the CLZ group spent a significantly larger proportion of time in remission than the $\mathrm{CPZ}$ group. Controlling for gender, baseline total BPRS, DUP, and age of onset of psychotic symptoms, the odds for being in remission was almost doubled for the CLZ group than the $\mathrm{CPZ}$ group (odds ratio $=1.73(1.20,2.50$ ), $p=0.003)$. The model also identified a significant effect for the DUP on remission status. With a 1-year increase in untreated psychosis, the odds of being in remission were reduced by nearly $15 \%$ (odds ratio $=0.85(0.75,0.95)$, $p=0.006)$.

$B P R S$ and other clinical measurements at the end of inpatient treatment. In the completer analysis, controlling for baseline, gender, DUP, and age of onset, patients who completed 12 weeks of inpatient treatment in the CLZ group experienced significantly greater reductions in psychopathology than $\mathrm{CPZ}$ on multiple rating scales including total BPRS, the BPRS Anergia Factor, total SANS, SANS Affective, Poverty of Speech, Avolition, and Anhedonia subscales, CGI severity and the GAF scales. In the LOCF analysis, CLZ evidenced significantly superior efficacy in the BPRS Anergia Factor, total SANS, SANS Affective and Avolition subscales, and was almost significant on the GAF scale (Table 1).
The analysis also found that patients with longer prior illness duration had higher total BPRS scores at 12 weeks $(\mathrm{F}(1,138)=6.83, p=0.01, \mathrm{OC})$ after controlling for their baseline scores, indicating a relation between DUP and therapeutic response. Similar findings supporting worse psychopathology with longer duration of untreated psychotic symptoms were found in other BPRS and SANS subscales (BPRS Anergia Factor $(\mathrm{F}(1,138)=8.02, p<0.01$, OC; $\mathrm{F}(1,154)=5.93, \quad p=0.02, \quad$ LOCF $), \quad$ total SANS $(\mathrm{F}(1,138)=6.95, p=0.01, \quad \mathrm{OC} ; \mathrm{F}(1,154)=8.55, p<0.01$, LOCF), SANS Avolition $(\mathrm{F}(1,154)=4.57, p=0.03$, LOCF), Anhedonia $(\mathrm{F}(1,138)=6.52, p<0.01, \mathrm{OC} ; \mathrm{F}(1,154)=8.46$, $p<0.01$, LOCF), and attention deficit $(\mathrm{F}(1,138)=17.01$, $p<0.01$, OC; $\mathrm{F}(1,154)=16.69, p<0.01, \mathrm{LOCF})$.

Since the major efficacy advantage of CLZ at 12 weeks was on negative symptom scores, to rule out the possibility that they were because of worse EPS in the CPZ group, we repeated the analysis of symptom reduction by treatment controlling for 12-week Parkinsonism severity. The changes in major findings were minimal. A significant efficacy advantage of CLZ over CPZ at week 12 was shown on BPRS total $(\mathrm{F}(1,137)=3.89 ; p=0.05)$, SANS total $(\mathrm{F}(1,137)=6.17$; $p=0.01)$, affective flattening $(\mathrm{F}(1,137)=10.52 ; p<0.01)$, avolition $(\mathrm{F}(1,137)=4.45 ; p=0.04)$, CGI $(\mathrm{F}(1,137)=5.48$; $p=0.02)$, and $\operatorname{GAF}(\mathrm{F}(1,137)=5.28 ; p=0.02)$ using $\mathrm{OC}$ population; and on BPRS anergy $(\mathrm{F}(1,153)=4.00 ; p=0.05)$ and SANS affective flattening $(\mathrm{F}(1,153)=10.48 ; p<0.01)$ with all ITT population using LOCF.

At 52 weeks there were no differences between the treatment groups on measurements of symptoms. Since the attrition rates were different in the two groups $(22.5 \%$ in $\mathrm{CPZ}$ and $15 \%$ in CLZ), an exploratory analysis was conducted that compared BPRS total scores at discharge between the patients who dropped out during follow up $v s$ those remaining on treatment. The dropouts in the $\mathrm{CPZ}$

Table I Efficacy Comparison for CLZ and CPZ at Weeks 12 and 52

\begin{tabular}{|c|c|c|c|c|c|c|c|c|c|c|}
\hline & \multirow{2}{*}{\multicolumn{2}{|c|}{$\begin{array}{c}\text { Baseline } \\
\text { Mean }^{\mathrm{a}}\end{array}$}} & \multicolumn{4}{|c|}{ I2 Weeks } & \multicolumn{3}{|c|}{52 Weeks } & \multirow[b]{3}{*}{$p^{b}$} \\
\hline & & & \multicolumn{2}{|c|}{ Mean $^{a}$} & \multirow{2}{*}{$\begin{array}{c}95 \% \mathrm{Cl}^{\mathrm{b}} \\
\text { Difference }\end{array}$} & \multirow[b]{2}{*}{$p^{b}$} & \multicolumn{2}{|c|}{ Mean $^{a}$} & \multirow{2}{*}{$\begin{array}{c}95 \% \mathrm{Cl}^{\mathrm{b}} \\
\text { Difference }\end{array}$} & \\
\hline & CPZ & CLZ & CPZ & CLZ & & & CPZ & CLZ & & \\
\hline \multicolumn{11}{|l|}{ BPRS } \\
\hline Total & 44.4 & 43.3 & 23.6 & 22.2 & $(-1.1,3.3)$ & 0.33 & 22.1 & 22.3 & $(-2.5,1.8)$ & 0.74 \\
\hline Anxiety/depression & 7.7 & 7.6 & 5.0 & 5.2 & $(-0.8,0.3)$ & 0.37 & 5.0 & 5.0 & $(-0.5,0.5)$ & 0.96 \\
\hline Anergy & 6.1 & 5.3 & 5.5 & 4.5 & $(0.2,1.3)$ & 0.01 & 4.9 & 4.6 & $(-0.5,0.7)$ & 0.68 \\
\hline Thought disorder & 13.4 & 13.6 & 5.7 & 5.3 & $(-0.5,1.2)$ & 0.40 & 5.1 & 5.2 & $(-1.0,0.7)$ & 0.74 \\
\hline Agitation/activation & 5.2 & 5.1 & 3.3 & 3.2 & $(-0.3,0.3)$ & 0.92 & 3.4 & 3.3 & $(-0.2,0.4)$ & 0.58 \\
\hline Hostility-paranoia & 12.0 & 11.7 & 4.1 & 4.0 & $(-0.6,0.8)$ & 0.71 & 3.8 & 4.2 & $(-1.1,0.3)$ & 0.23 \\
\hline \multicolumn{11}{|l|}{ SANS } \\
\hline Total & 16.6 & 14.8 & 11.4 & 6.9 & $(1.0,7.0)$ & 0.01 & 9.5 & 7.5 & $(-1.9,4.7)$ & 0.40 \\
\hline Affective flattening & 2.2 & 0.9 & 3.3 & 1.0 & $(1.1,3.1)$ & $<0.01$ & 2.2 & 1.0 & $(-0.0,2.0)$ & 0.05 \\
\hline Poverty of thought & 1.4 & 0.6 & 1.0 & 0.5 & $(-0.1,0.8)$ & 0.10 & 0.7 & 0.4 & $(-0.3,0.7)$ & 0.44 \\
\hline Avolition & 7.1 & 7.0 & 3.7 & 2.6 & $(0.0,2.0)$ & 0.05 & 3.5 & 3.0 & $(-0.6,1.5)$ & 0.40 \\
\hline Attention deficit & 0.6 & 0.6 & 0.6 & 0.4 & $(-0.3,0.6)$ & 0.48 & 0.4 & 0.3 & $(-0.3,0.5)$ & 0.54 \\
\hline Low level of interests & 5.4 & 5.6 & 3.0 & 2.4 & $(-0.5,1.5)$ & 0.30 & 2.7 & 2.8 & $(-1.3,1.0)$ & 0.80 \\
\hline CGl & 5.6 & 5.6 & 2.4 & 2.1 & $(-0.1,0.7)$ & 0.13 & 2.0 & 2.2 & $(-0.6,0.2)$ & 0.36 \\
\hline GAF & 35.4 & 36.2 & 67.7 & 72.8 & $(-9.7,0.2)$ & 0.06 & 71.4 & 72.4 & $(-5.7,4.8)$ & 0.86 \\
\hline
\end{tabular}

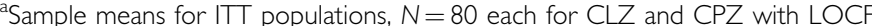

${ }^{b}$ By ANCOVA model with LOCF adjusted for gender, baseline severity, age of first onset, and duration of psychotic symptoms. Analysis with $O C$ replicated all the significant findings with LOCF at 12 weeks. It showed that CLZ significantly outperformed CPZ in several other scales, including total BPRS $(p=0.03)$, SANS poverty of thought $(p=0.03)$, avolition $(p<0.0 \mathrm{I})$, low level of interest $(p=0.03), \mathrm{CGI}(p=0.0 \mathrm{I})$, and GAF $(p<0.0 \mathrm{I})$. 
group had significantly higher BPRS scores (26.0[7.5]) at discharge than the other three groups (22.1[5.0] for CPZ ontreatment, 20.6[4.1] for CLZ on-treatment, and 20.3[2.5] for CLZ dropouts). The dropout of sicker patients on CPZ may have contributed to the lack of a persistent difference between the two groups in the LOCF analysis at 52 weeks.

There were 14 rehospitalizations in weeks 13-52. Five patients on CPZ had a total of seven rehospitalizations while six patients on CLZ had seven rehospitalizations. There were no known suicide attempts by any study participants in the 1 st year of the study.

\section{Safety}

Extrapyramidal symptoms. The means for the total SAESS and Parkinsonism are listed in Table 2. Despite the use of prophylactic BZ, the $\mathrm{CPZ}$ group consistently showed more EPS than the CLZ group. At both weeks 12 and 52, patients receiving $\mathrm{CPZ}$ had significantly higher total scores on the Simpson Angus Scale and the Parkinsonism subscale with OC analysis. The LOCF analysis corroborated the significant difference at 12 weeks, but the 52 weeks comparison was again not significant. The ANCOVA model also found that females had more severe Parkinsonism than males at week $12(\mathrm{~F}(1,138)=5.51, \quad p=0.02, \quad \mathrm{OC}$; $\mathrm{F}(1,154)=5.30, p=0.02$, LOCF $)$, but the difference was not significant at week 52 .

Other side effects. Individual adverse events with significant treatment effects are listed in Table 3. Over the course of study follow up, the CLZ treatment group was at a significantly lower risk for developing side effect symptoms such as dystonia, blurred vision, muscle tension, dry mouth, akathisia, objectively observed restlessness, and decreased urine production, and showed an increased risk for sweating and a marginally increased risk for depressed affect. As stated earlier, eight patients were withdrawn because of adverse effects; six from CPZ (constipation, hyperthermia, ileus, dermatitis, neutropenia, EPS) and two from CLZ (hyperthermia, hypotension). One patient (35year-old male) died at week 10 of treatment with $\mathrm{CPZ}$ while in the hospital after developing hyperthermia $(41.5 \mathrm{C})$ and leukocytosis (16300 with $78 \%$ neutrophils) but no rigidity or marked alteration of consciousness. The cause of death was determined to be hyperthermia possibly because of viral encephalitis. (This event occurred in summer during a heat wave in Beijing.)

At study entry the CLZ and CPZ groups had similar total WBC (7887[2270]), percentages of polymorphonuclear neutrophiles $(64 \%[8 \%])$ and lymphocytes $(36 \%[8 \%])$, fasting glucose levels $(4.7[0.96] \mathrm{mmol} / \mathrm{l})$, heart rates $(81[17.3])$, and QT intervals $(0.35[0.04] \mathrm{ms})$ (Table 4). After treatment

Table 2 Simpson-Angus Extrapyramidal Symptom Scale (SAESS) Total Score and Parkinsonian Score for CLZ and CPZ at Weeks 12 and $52^{\mathrm{a}}$

\begin{tabular}{|c|c|c|c|c|}
\hline & \multicolumn{2}{|c|}{ Mean } & \multirow{2}{*}{$\begin{array}{c}95 \% \mathrm{Cl}^{\mathrm{b}} \\
\text { Difference }\end{array}$} & \multirow[b]{2}{*}{$p^{b}$} \\
\hline & CPZ & CLZ & & \\
\hline \multicolumn{5}{|l|}{12 Weeks } \\
\hline SAESS total & 1.41 & 0.41 & $(0.47,1.38)$ & $<\mathbf{0 . 0 1}$ \\
\hline Parkinsonian & 0.90 & 0.38 & $(0.14,0.75)$ & $<\mathbf{0 . 0 1}$ \\
\hline \multicolumn{5}{|l|}{52 Weeks } \\
\hline SAESS total & 0.44 & 0.28 & $(-0.18,0.44)$ & 0.40 \\
\hline Parkinsonian & 0.33 & 0.18 & $(-0.11,0.32)$ & 0.32 \\
\hline
\end{tabular}

aITT population, $N=80$ in each of the CPZ and CLZ groups, LOCF. Analysis using $O C$ replicated the significant 12 weeks finding. In addition, the differences at 52 weeks remained significant using $O C(p=0.04$ and 0.02 for SAESS and Parkinsonian, respectively).

${ }^{\text {b} A d j u s t e d ~ f o r ~ g e n d e r, ~ b a s e l i n e ~ s e v e r i t y, ~ D U P ~ a n d ~ a g e ~ o f ~ o n s e t ~ i n ~ A N C O V A ~}$ model.

Table 3 Significant Treatment Effects (CLZ vs CPZ) on Occurrence of Individual Adverse Events During Follow-Up (up to 52 weeks) ${ }^{a}$

\begin{tabular}{|c|c|c|c|c|c|}
\hline \multirow[b]{2}{*}{ Adverse event } & \multicolumn{2}{|c|}{ Mean risk ${ }^{\mathbf{b}}$} & \multirow[b]{2}{*}{$O^{c}$} & \multirow[b]{2}{*}{$(95 \% \mathrm{Cl})$} & \multirow[b]{2}{*}{$p$} \\
\hline & CPZ & CLZ & & & \\
\hline \multicolumn{6}{|l|}{ SAESS } \\
\hline Dystonia & 0.11 & 0.07 & 0.24 & $(0.10,0.57)$ & 0.0013 \\
\hline \multicolumn{6}{|l|}{ Side effects } \\
\hline Blurred vision & 0.46 & 0.33 & 0.53 & $(0.38,0.74)$ & 0.0002 \\
\hline Tense muscles & 0.08 & 0.06 & 0.33 & $(0.12,0.87)$ & 0.0250 \\
\hline Depressed affect & 0.19 & 0.25 & 1.41 & $(1.00,2.05)$ & 0.0500 \\
\hline Sweating & 0.06 & 0.11 & 3.04 & $(1.51,6.10)$ & 0.0018 \\
\hline Dry mouth & 0.64 & 0.32 & 0.22 & $(0.17,0.30)$ & $<0.000$ I \\
\hline Akathisia & 0.13 & 0.09 & 0.47 & $(0.26,0.83)$ & 0.0098 \\
\hline Objectively observed restlessness & 0.09 & 0.06 & 0.39 & $(0.18,0.85)$ & 0.0171 \\
\hline Decreased urine production & 0.12 & 0.07 & 0.23 & $(0.11,0.47)$ & $<0.0001$ \\
\hline
\end{tabular}

aITT population, $N=80$ each for $C P Z$ and $C L Z$. Other adverse events tested but nonsignificant include SAESS akathisia, weakness, increased/decreased appetite, disorganized thinking, constipation, low libido, dermatitis, diarrhea, light headed, insomnia, nocturnal enuresis, agitate, dizzy, tired, headache, dysmenorhrea, muscular spasms, nausea, drowsiness, sleep problems, sinning sensation, polyuria, daytime enuresis, and feebleness.

bample mean for the proportion of visits recorded with the adverse event.

'Odds ratios after controlling for patient sex, duration of psychosis, and age of onset. Numbers less than I indicate that the CLZ group is less likely to experience the side effect than the CPZ group and vice versa. 
Table 4 Laboratory and other Clinical Parameters at Weeks 12 and 52

\begin{tabular}{|c|c|c|c|c|}
\hline & \multicolumn{2}{|c|}{ Mean $^{a}$} & \multirow{2}{*}{$\begin{array}{c}95 \% \mathrm{Cl}^{\mathrm{b}} \\
\text { Difference }\end{array}$} & \multirow[b]{2}{*}{$p^{b}$} \\
\hline & CPZ & CLZ & & \\
\hline \multicolumn{5}{|l|}{ Baseline } \\
\hline Neutrophils (\%) & 65.2 & 63.0 & & \\
\hline Lymphocytes (\%) & 34.6 & 36.7 & & \\
\hline WBC & 7798 & 7977 & & \\
\hline Glucose $(\mathrm{mmol} / \mathrm{l})$ & 4.8 & 4.7 & & \\
\hline EKG heart rate ${ }^{c}$ & 81.9 & 80.0 & & \\
\hline EKG QT interval ${ }^{c}$ & 0.35 & 0.35 & & \\
\hline \multicolumn{5}{|l|}{12 Weeks } \\
\hline Neutrophils (\%) & 66.7 & 64.8 & $(-0.2,4.4)$ & 0.08 \\
\hline Lymphocytes (\%) & 33.3 & 34.9 & $(-4.1,0.5)$ & 0.13 \\
\hline WBC & 8368 & 8331 & $(-568,770)$ & 0.77 \\
\hline Glucose $(\mathrm{mmol} / \mathrm{l})$ & 4.9 & 5.1 & $(-0.69,0.12)$ & 0.16 \\
\hline EKG heart rate ${ }^{c}$ & 86.6 & 90.8 & $(-8.6,0.9)$ & 0.11 \\
\hline EKG QT interval ${ }^{c}$ & 0.36 & 0.34 & $(0.01,0.03)$ & $<0.01$ \\
\hline \multicolumn{5}{|l|}{52 Weeks } \\
\hline Neutrophils (\%) & 66.3 & 67.2 & $(-2.8,||)$. & 0.40 \\
\hline Lymphocytes (\%) & 33.7 & 32.7 & $(-1.0,2.9)$ & 0.34 \\
\hline WBC & 7954 & 8668 & $(-1318,23)$ & 0.06 \\
\hline Glucose $(\mathrm{mmol} / \mathrm{l})$ & 5.5 & 5.3 & $(-0.32,0.67)$ & 0.49 \\
\hline EKG heart rate ${ }^{c}$ & 88.7 & 94.1 & $(-\mid 1.1,-1.5)$ & 0.01 \\
\hline EKG QT interval ${ }^{c}$ & 0.34 & 0.33 & $(0.01,0.02)$ & $<\mathbf{0 . 0 1}$ \\
\hline
\end{tabular}

there were no significant differences between the two drugs for total WBC or glucose level. OC analysis found a lower percent of neutrophiles and higher lymphocytes at week 12 for patients taking CLZ $(\mathrm{F}(1,102)=5.75, p=0.02$ and $\mathrm{F}(1,102)=5.12, p=0.03$, respectively), but it was not supported by the LOCF analysis, nor were the significant findings maintained at week 52 by OC or LOCF analysis. The CLZ group had a significantly shorter QT interval at both 12 weeks $(\mathrm{F}(1,126)=16.61, p<0.001$, OC; $\mathrm{F}(1,143)=15.64, \quad p<0.001, \quad$ LOCF $) \quad$ and 52 weeks $(\mathrm{F}(1,143)=10.25, p=0.002, \mathrm{LOCF})$, and a higher heart rate at 52 weeks $(\mathrm{F}(1,60)=6.88, p=0.01, \mathrm{OC} ; \mathrm{F}(1,149)=6.70$, $p=0.01, \mathrm{LOCF})$.

Weight. Baseline and follow-up weights were obtained for $72 \%$ of subjects ( $71 \%$ for $\mathrm{CPZ}$ and $73 \%$ for CLZ). The median times for the first weight measurement during follow-up were 131 weeks after starting medication for CPZ vs 137.5 weeks after starting medication for CLZ. For the available data, the mean weights were $60.3 \mathrm{~kg}(9.7)$ for $\mathrm{CPZ}$ and $61.6 \mathrm{~kg}(10.1)$ for CLZ at baseline; and $68.8 \mathrm{~kg}(12.0)$ and $71.5 \mathrm{~kg}$ (13.3) for CPZ vs CLZ at the first follow-up, which was significant by an ANCOVA model of weight change (controlling for gender, baseline weight, age of first onset and duration of psychotic symptoms and follow-up); weight increases from baseline to follow-up $(\mathrm{F}(1,108)=36.6$, $p<0.01)$. However, the difference in the mean weight gain for CPZ $(6.5 \mathrm{~kg})$ and CLZ $(9.9 \mathrm{~kg})$ was not statistically significant $(\mathrm{F}(1,108)=1.10, p=0.30)$.

\section{DISCUSSION}

This study is the first to examine the long-term outcome of treatment-naive first-episode schizophrenia patients in response to atypical and conventional antipsychotic drugs in the context of a randomized double-blind controlled trial. These results are consistent with prior studies in Western countries, which demonstrated that first-episode patients are capable of having good therapeutic responses to antipsychotic medications (Lieberman et al, 1993; Sheitman et al, 1997; Robinson et al, 1999). Moreover, the demographic and clinical characteristics of the sample, including the duration of prior psychotic symptoms, were comparable to those of patients in prior studies from Western countries further indicating the generalizability of these findings (Sanger et al, 1999; Crow et al, 1986; Loebel et al, 1992; Johannesen et al, 2001). The slightly later age of onset reported by patients in this study (mean 27.2 years) relative to that reported in Western countries is an interesting finding that deserves further investigation. The relation between increased duration of psychosis with poorer treatment outcome is consistent with most (Loebel et al, 1992; Wyatt, 1999; Szymanski et al, 1996) but not all (BengChoon et al, 2000; Craig et al, 2000; Barnes et al, 2000) prior studies. The study's results also suggest that CLZ, the prototype of atypical drugs, may have superior benefits to conventional medications reflected by faster treatment responses, significantly improved better short-term efficacy, more time spent in remission, reduced EPS and other 
adverse side effects, and better retention of patients in treatment. The prophylactic use of $4 \mathrm{mg}$ per day of $\mathrm{BZ}$ in the $\mathrm{CPZ}$ group may have contributed to some of the anticholinergic side effects. At the same time, CLZ was associated with more sweating and increased heart rates. Sialorrhea was not assessed in this study but is known to be common with CLZ use. In spite of the previously reported association of CLZ and other atypical antipsychotic drugs on glucose metabolism (Henderson et al, 2000), there were no significant differences between treatments in this trial. Although weights were not systematically obtained, both CPZ and CLZ were associated with substantial weight gain consistent with previous studies (Allison et al, 1999). Cholesterol and triglyceride elevations, which have also been associated with CLZ treatment, were not assessed (Henderson, 2000). CLZ -treated patients had higher heart rates, but this did not cause any impairment to patients or lead to treatment discontinuations.

Although the rapidity and amount of time in remission was greater for CLZ, the two drugs did not differ on symptom ratings at 52 weeks. The high degree of treatment responsiveness of patients early in the course of schizophrenia may make the demonstration of differences in treatments on the long-term outcomes using traditional measures of psychopathology difficult. Despite the potential 'ceiling effect', CLZ produced significantly better symptom reductions than $\mathrm{CPZ}$ at 12 weeks. The failure to sustain the significant differences in symptom reduction between CLZ and CPZ beyond 12 weeks could have been because of more posthospitalization dropouts among less responsive patients in the CPZ group than the CLZ group, thereby decreasing the differences in improvement seen at 52 weeks as suggested by the exploratory analysis. Thus, long-term follow-up of all patients who entered the study including those who dropped out would be informative. In addition, it may be necessary to employ additional measures of outcome beyond symptom ratings to characterize more fully the treatment effects of antipsychotic drugs and discern the potential advantages of CLZ as representative of the atypicals. However, it is also debatable to what extent the effects of CLZ in this patient population would generalize to the other atypical agents. If, as these results suggest, atypical drugs produce faster rates of symptom remission, cause fewer side effects, and are associated with better treatment adherence compared to conventional drugs, then follow-up studies longer than 1 year may be necessary to demonstrate putative benefits reflected by reduced relapse rates, illness progression, changes in cognitive functions, and cumulative morbidity. The full extent of the potential superiority in treatment effects of the atypical antipsychotic drugs may only be appreciated in the context of very long-term outcome studies or after extensive clinical experience with these compounds in a new generation of patients who receive them from the beginning of their illness.

\section{ACKNOWLEDGMENTS}

We acknowledge the assistance of the following staff members at Beijing Hui Long Guan Hospital in carrying out this project: (alphabetically listed) Peiran Guo, Feifei Li,
Guowang Li (Deceased), Huaqing Li, Lanying Li, Xianyun Li, Mei Meng, Yajuan Niu, Lan Shang, Jiancheng Song, Deliang Yan, Fude Yang, Xiuqin Zhao, and Fengyan Zhu. The investigators appreciate the support and encouragement of Drs Peter Jager, Rajinder Judge, Jeffrey Schwimmer, and Rene Speigel of Novartis in the development and implementation of the study. This work was supported by USPHS grants MH00537, MH33127 (Dr Lieberman), the UNC Mental Health and Neuroscience Clinical Research Center, and the Novartis Pharmaceutical Company.

\section{REFERENCES}

Allison DB, Mentore JL, Heo M, Chandler LP, Cappelleri JC, Infante MC et al (1999). Antipsychotic-induced weight gain: a comprehensive research synthesis. Am J Psychiatry 156: 16861696.

Barnes TR, Hutton SB, Chapman MJ, Mutsatsa S, Puri BK, Joyce EM (2000). West London first-episode study of schizophrenia: clinical correlates of duration of untreated psychosis. $\mathrm{Br} J$ Psychiatry 177: 207-211.

Beng-Choon H, Andreassen NC, Flaum M, Nopoulos P, Miller D (2000). Untreated initial psychosis: its relation to quality of life and symptom remission in first episode schizophrenia. Am J Psychiatry 157: 808-815.

Chakos MH, Alvir JMJ, Woerner MG, Koreen A, Geisler S, Mayerhoff D et al (1996). Incidence and correlates of tardive dyskinesia in first episode of schizophrenia. Arch Gen Psychiatry 53: 313-319.

Chakos M, Lieberman JA, Hoffman E, Bradford D, Sheitman B (2001). Effectiveness of second-generation antipsychotics in patients with treatment-resistant schizophrenia: a review and meta-analysis of randomized trials. Am J Psychiatry 158: 518-526.

Chatterjee A, Chakos M, Koreen A, Geisler S, Sheitman B, Alvir J et al (1995). Prevalence and clinical correlates of extrapyramidal signs and spontaneous dyskinesias in never medicated schizophrenic patients. Am J Psychiatry 152: 1724-1729.

Craig TJ, Bromet EJ, Fennig S, Tanenberg-Karant M, Lavelle J, Galambos N (2000). Is there an association between duration of untreated psychosis and 24 month clinical outcome in a first admission series? Am J Psychiatry 157: 60-66.

Crow TJ, MacMillan JF, Johnson AL, Johnstone EC (1986). A randomized controlled trial of prophylactic neuroleptic treatment. Br J Psychiatry 148: 120-127.

Emsley RA (1999). Risperidone in the treatment of first-episode psychotic patients: a double-blind multicenter study. Schizophr Bull 25: 721-729.

Geddes J, Freemantle N, Bebbington P (2000). Atypical antipsychotics in the treatment of schizophrenia: systematic overview and meta-regression analysis. $\mathrm{Br}$ Med J 321: 1360-1361.

Hegarty JD, Baldessarini RJ, Tohen M, Waternaux C, Oepen G (1994). One hundred years of schizophrenia: a meta-analysis of the outcome literature. Am J Psychiatry 151: 1409-1416.

Henderson DC, Cagliero E, Gray C, Nasrallah RA, Hayden DL, Schoenfeld DA et al (2000). Clozapine, diabetes mellitus, weight gain and lipid abnormalities: a five year naturalistic study. Am J Psychiatry 157: 975-981.

Herz MI, Liberman RP, Lieberman JA, Marder SR, McGlashan TH, Wyatt RJ et al (1997). Practice guidelines for the treatment of patients with schizophrenia. Am J Psychiatry 154(Suppl): 4.

Johannesen JO, McGlashan TH, Larsen TK, Horneland M, Joa I, Mardal S et al (2001). Early detection strategies for untreated first-episode psychosis. Schizophr Res 51: 39-46.

Kinon BJ, Lieberman JA (1996). Mechanisms of action of atypical antipsychotic drugs: a critical analysis. Psychopharmacology 124: $2-34$. 
Kraeplin E (1919). Dementia Praecox and Paraphrenia. E\&S Livingstone: Edinburgh, Scotland.

Leucht S, Pitschel-Walz G, Abraham D, Kissling W (1999). Efficacy and extrapyramidal side-effects of the new antipsychotics olanzapine, quetiapine, risperidone, and sertindole compared to conventional antipsychotics and placebo. Schizophr Res 35: 51-68.

Lieberman J, Jody D, Geisler S, Alvir J, Loebel A, Szymanski S et al (1993). Time course and biologic correlates of treatment response in first-episode schizophrenia. Arch Gen Psychiatry 50: 369-376.

Lieberman JA (1996). Atypical antipsychotic drugs as a first-line treatment of schizophrenia: a rationale and hypothesis. J Clin Psychiatry 57(Suppl 11): 68-71.

Lieberman JA, Sheitman B, Chakos M, Robinson D, Schooler N, Keith S (1998). The development of treatment resistance in patients with schizophrenia: a clinical and pathophysiological perspective. J Clin Psychopharmacol 18: S20-S24.

Loebel AD, Lieberman JA, Alvir JMJ, Mayerhoff DI, Geisler SH, Szymanski SR (1992). Duration of psychosis and outcome in first-episode schizophrenia. Am J Psychiatry 149: 1183-1188.

McEvoy JP, Hogarty GE, Steingard S (1991). Optimal dose of neuroleptic in acute schizophrenia. Arch Gen Psychiatry 48: 739745.

McGlashan TH (1988). A selective review of recent North American long-term follow-up studies of schizophrenia. Schizophr Bull 14: 515-542.

Norman RM, Malla AK (2001). Duration of untreated psychosis: a critical examination of the concept and its importance. Psychol Med 31: 381-400.
Phillips M (2001). Characteristics, experience and treatment of schizophrenia in China. Dialog Clin Neurosci 3: 109-119.

Phillips MR, Xiong W, Wang RW, Gao RH, Wang XQ, Zhang NP (1991). Reliability and validity of the Chinese versions of the scales for assessment of positive and negative symptoms. Acta Psychiatr Scand 84: 364-370.

Phillips MR, Xiong W, Zhao ZA (1990). Issues Involved in the Use of Scales for the Assessment of Negative and Positive Symptoms in Psychotic Patients. Hubei Science and Technology Publishing House: Wuhan, PRC (in Chinese).

Robinson D, Woerner M, Alvir J, Bilder R, Goldman R, Geisler S et al (1999). Predictors of treatment response from a first episode of schizophrenia or schizoaffective disorder. Am J Psychiatry 156: $544-549$.

Sanger TM, Lieberman JA, Tohen M, Grundy S, Beasley C, Tollefson GD (1999). Olanzapine versus haloperidol treatment in first-episode psychosis. Am J Psychiatry 156: 79-87.

Sheitman BB, Lee H, Strous R, Lieberman JA (1997). The evaluation and treatment of first episode psychoses. Schizophr Bull 23: 653-661.

Szymanski SR, Cannon TD, Gallacher F, Erwin RJ, Gur RE (1996). Course and treatment response in first-episode and chronic schizophrenia. Am J Psychiatry 153: 519-525.

Wu B, Wu JP (1995). Investigation of antipsychotic use in hospitalized patients. Appl Psychiatry 2: 19-22 (in Chinese).

Wyatt RJ (1999). Early intervention with neuroleptcis may decrease the long-term morbidity of schizophrenia. Schizophr Res 5: 201-202. 